\title{
Verification of Focusing from a Hemispherically Shaped Surface.
}

\author{
Benjamin L. Brown, Tamara S. Andrew, \\ Margaret S. Clarkson, C. Sean Sutton \\ Mount Holyoke College \\ South Hadley, MA 01075 \\ Pedro Encarnación \\ University of Michigan \\ Ann Arbor, MI \\ Art Denison and Henry Makowitz, \\ EG\&G INEL \\ Idaho Falls, ID \\ Kermit Bundy \\ Idaho State University \\ Pocatello, ID
}

\begin{abstract}
The proposed intense slow positron source (ISPS)' at Idaho National Engineering Laboratory (INEL) requires focusing from a large area source to a small $(1 \mathrm{~cm})$ remoderator foil. A diameter reduction of $>30$ is desirable, and a gridless design appears to accommodate the design requirements. Experimental confirmation of the gridless focusing concept is presented here together with SIMION (simulated ion tracking) computer simulations, which indicate a diameter reduction of $\sim 50 x$.
\end{abstract}

Prepared for U. S. Department of Energy through the EG\&G Idaho, Inc. LDRD program. Under DOE-ID Contract DE-AC07-76ID01570 
The proposed intense slow positron source at INEL will have a maximum of $10^{13}$ slow positrons emanating from a large area source dish. In order to focus the positrons efficiently to the foil remoderator for brightness enhancement, an appropriately designed electrostatic focusing geometry has been studied. The design features gridless focusing, thus avoiding the complexity and inefficiency of grid optics, and making the experimental goal of a $>30 x$ diameter reduction much easier.

The SIMION program uses relaxation calculations to obtain solutions to Laplace's equation with specified boundary conditions, and the potentials in our case are calculated to $\sim 1 \%$ accuracy. In addition, an analytical solution to a nearly identical geometry indicates agreement with both the SIMION simulations and the PMT measurements. A separate measurement of a uniformly illuminated photocathode shows linearity well above a total current of $1 \mu \mathrm{a}$, indicating no unforeseen problem at the anticipated positron beam currents.

After some initial design considerations involving a grid focused system, ${ }^{2,3}$ a gridless design appears to be superior for the ISPS beam. The gridless design has the following advantages: increased efficiency $(x 2)$ with the grid and grid mounting structure eliminated; increased focusing possible due to the elimination of grids that can produce significant defocusing; total insensitivity of alignment in the gridless geometry as opposed to the very fine tolerances and external adjustments needed in the grid focused beam; easy adaptability to a cryogenic gas moderator system; less sensitivity to surface roughness than the grid case; and ease of construction. A typical hemispherical configuration for focusing is shown in Fig. 1. The radius of the hemisphere is $25 \mathrm{~cm}$. The positrons are attracted to a central disk that is biased at $-5 \mathrm{kV}$, relative to all other surfaces which are at or near ground potential.

A photomultiplier tube manufactured by Hammamatsu (Fig. 2) has a geometry similar to the geometry that we wish to use with the ISPS at INEL (Fig.3). In the work presented here, we analyze the focusing of low energy photoelectrons from the hemispherical surface of the photomultiplier. All measurements were taken with the PMT in a light tight box with a single green LED as a light source. The LED is positioned externally. The photon count rate was recorded, as the distance from the LED to the PMT was varied both laterally (A) and longitudinally (Z). The measurements show, with a small correction for a systematic variation with position $Z$, the distance of the LED from the PMT, there is no significant variation in the photon count as a function of arc distance (A in cm) from the central axis. Refer to Fig. 4. This demonstrates a diameter reduction of 8:1 for the first stage, limited only by the large $(2.5 \mathrm{~cm})$ acceptance diameter of the first dynode stage.

A SIMION simulation of the PMT geometry is shown in Fig. 3. The 


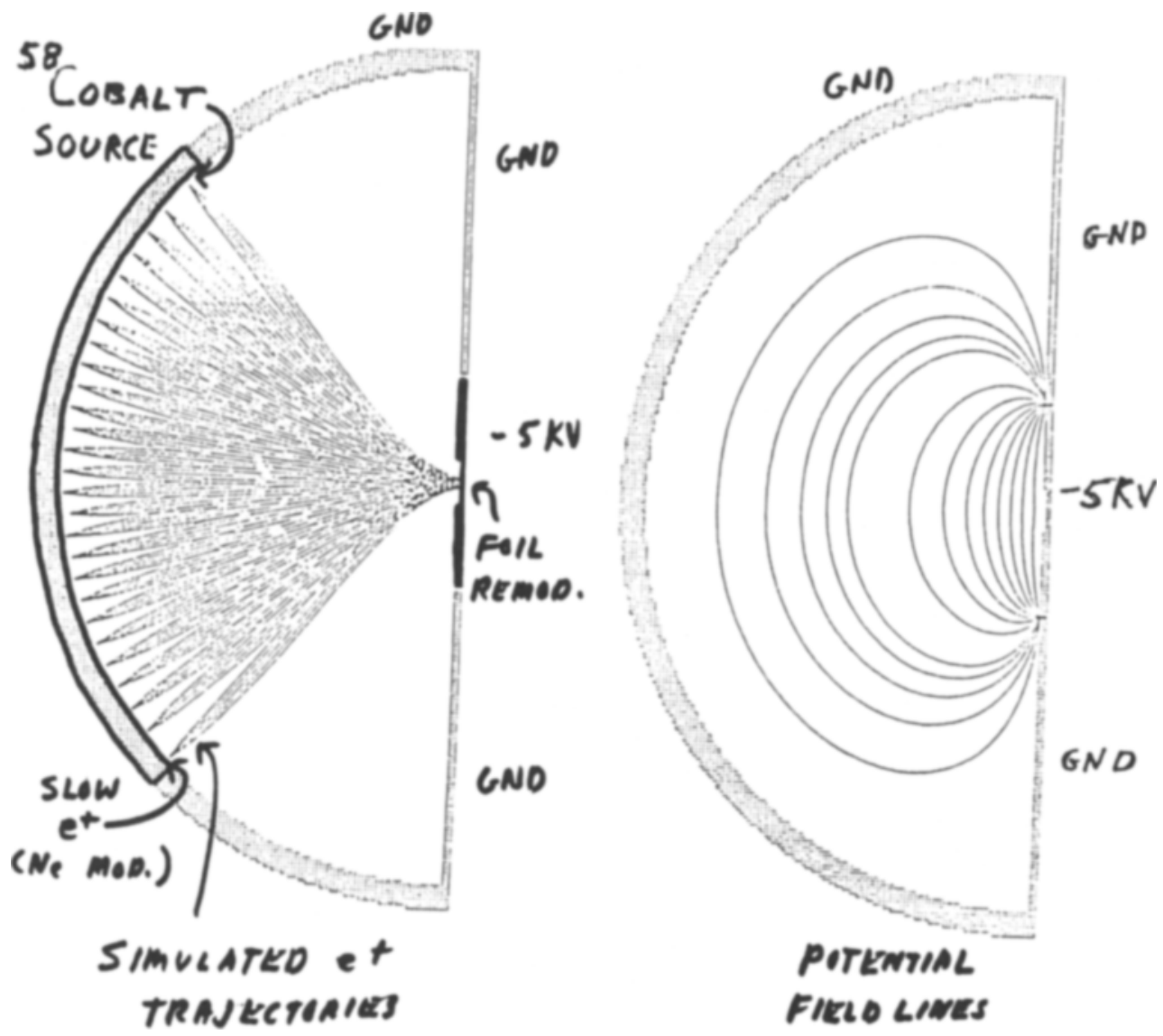

FIGURE 1. Simion simulation of the first stage of an intense positron beam source (ISPS) proposed to be built at INEL. The positron trajectories are shown on the left, with positrons launched at $1 \mathrm{eV}$ with an angle varying to 45 degrees from the surface. Equipotential curves are also shown on the right. The surface shown is a hemisphere which mates to an annular plate. A small disk at the center of the plate is biased to $-5 \mathrm{kV}$. The scale of the proposed beam corresponds to a $25 \mathrm{~cm}$ radius of the hemisphere. 
internal structure of the tube is shown and the figure represents a cross section with cylindrical symmetry. A second dynode voltage of 800 volts was applied, followed by a multi-dynode chain for amplification (not shown) located in the grounded central cylinder. The focusing in the simulation appears to be dramatic, and it corresponds to the experimental PMT results. A trick was used in the SIMION simulation, and in most of our other similar simulations, to minimize the rough surface effects due to the finite mesh at the hemispherical boundary. The trick is the following: the positrons were launched along an equipotential surface a few mesh units away from the rough surface where the potentials are likewise rougher. This gives a much smoother hemispherical potential surface from which to launch the positrons. The smoothness is easily attainable in practice. With the gridless focusing, the trick improves the focusing slightly ( $<10 \%$ with the finest mesh size used). In simulations of some grid focusing geometries where a grid is placed very near the hemispherical surface, ${ }^{3}$ the trick improves the simulation focusing dramatically ( $\mathrm{x} 2$ or more). In grid designs where the grid is nearer the target and farther from the hemisphere, ${ }^{2,4}$ the design is less sensitive to surface roughness and alignment considerations. The trick does not improve the simulation focusing as much for this design.

An analytical solution which is also close to the current geometry appears to independently confirm the above results. Using a Bessel function solution to Laplace's equation, it is not difficult to solve the problem with the boundary conditions of a biased disk set into an infinite grounded plane. ${ }^{5}$ This problem is substantially similar to our proposed geometry. From the Bessel function solution involving the potential, the $E$ field can be obtained from the gradient of the potential, and the characteristic focusing determined. Positrons are assumed to be launched on an equipotential surface which approximates our hemispherical surface of emission at a distance of several disk radii from the disk center. The hemispherical emission surface is thus at a slightly more negative potential (for positrons) than the ground potential. This equipotential surface can be correspondingly biased in our SIMION calculations (biasing the hemisphere slightly negative in Fig. 1 for example) for comparison to the Bessel function solution. The analytical solution further supports the simulations and the PMT measurements.

Calculations of space charge effects show no significant effects in the positron beam current range of $1 \mu \mathrm{A}\left(\sim 10^{13} \mathrm{e}^{+} / \mathrm{s}\right)$, which represents a static density of $\sim 100 \mathrm{e}^{+} / \mathrm{cm}^{3}$. We also examined the Vlassov equation and determined that the E field external, is $>10^{5} \mathrm{x}$ the induced $\mathrm{E}$ field, and thus collective effects in transport are negligible. Indeed, the plasma oscillation time is an order of magnitude larger than the transit time from the dish to the remoderator. Possible charging of the Ne moderator must be investigated in detailed tests using an intense source. 
204 Verification of Focusing

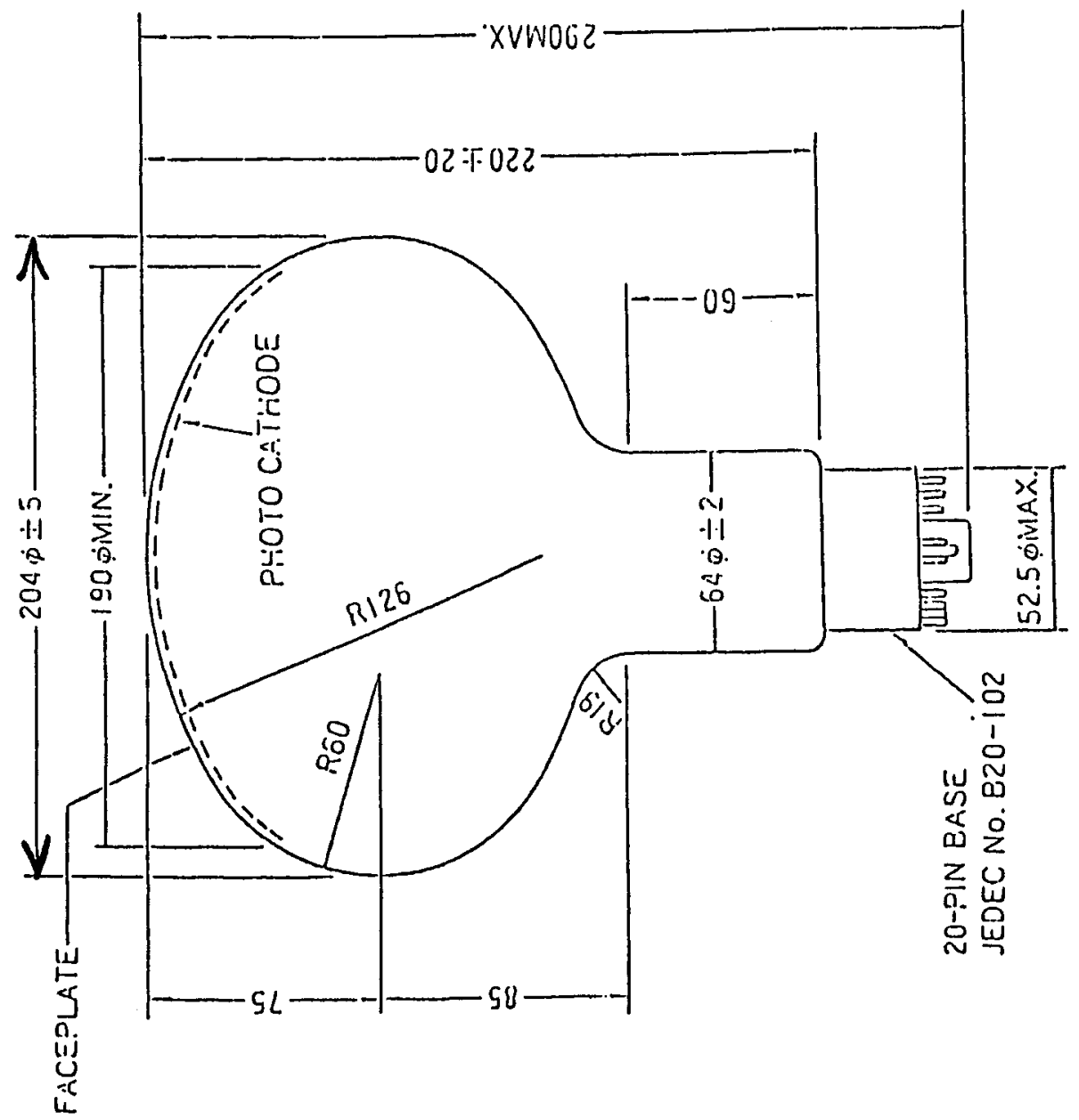

FIGURE 2. Hammamatsu phototube R-1408. This tube uses the same geometry for focusing as the proposed ISPS beam. 
B. L. Brown et al. 205

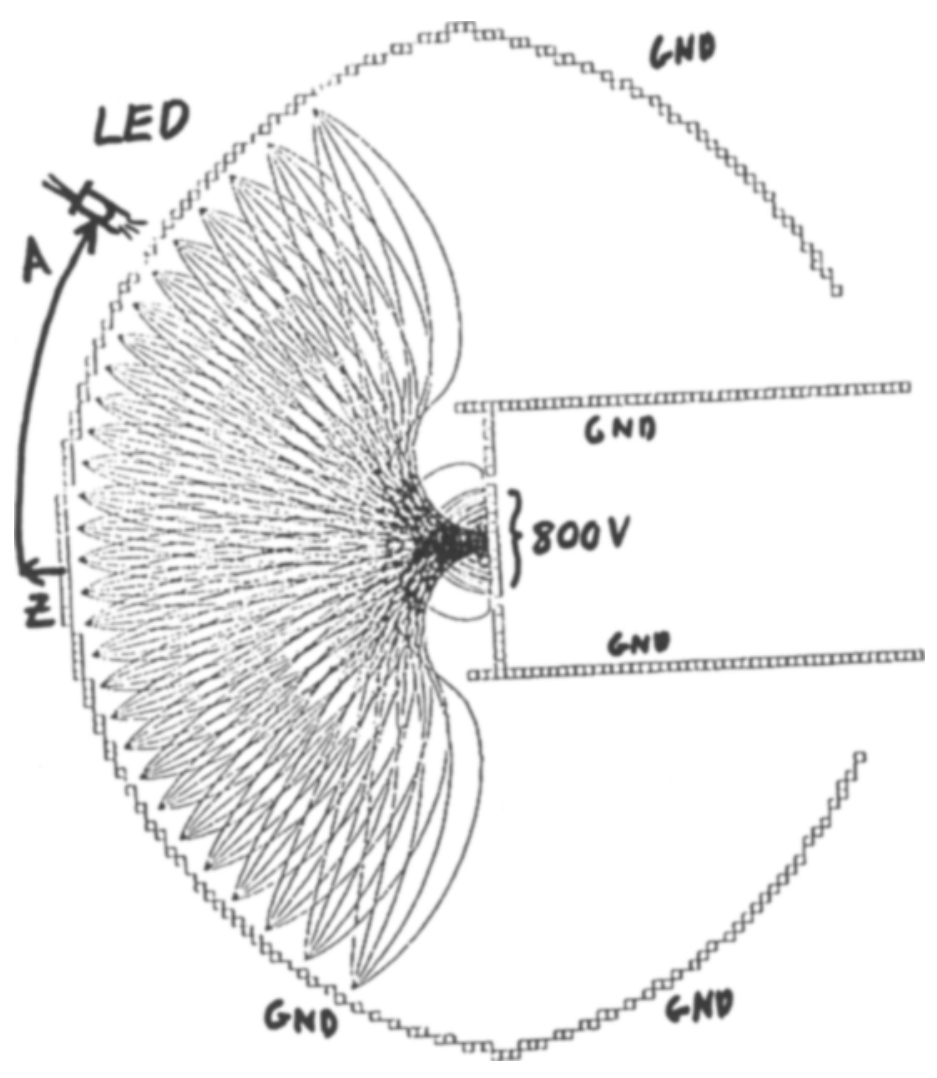

FIGURE 3. SIMION simulation of the PMT focusing from $\sim 20 \mathrm{~cm}$ to $\sim 1$ $\mathrm{cm}$. The electrons at extreme angles to the central axis (large values of $\mathrm{A}$ ) tend to fall outside the central $0.6 \mathrm{~cm}$ spot. 


\section{COUNTS/S VS. A}

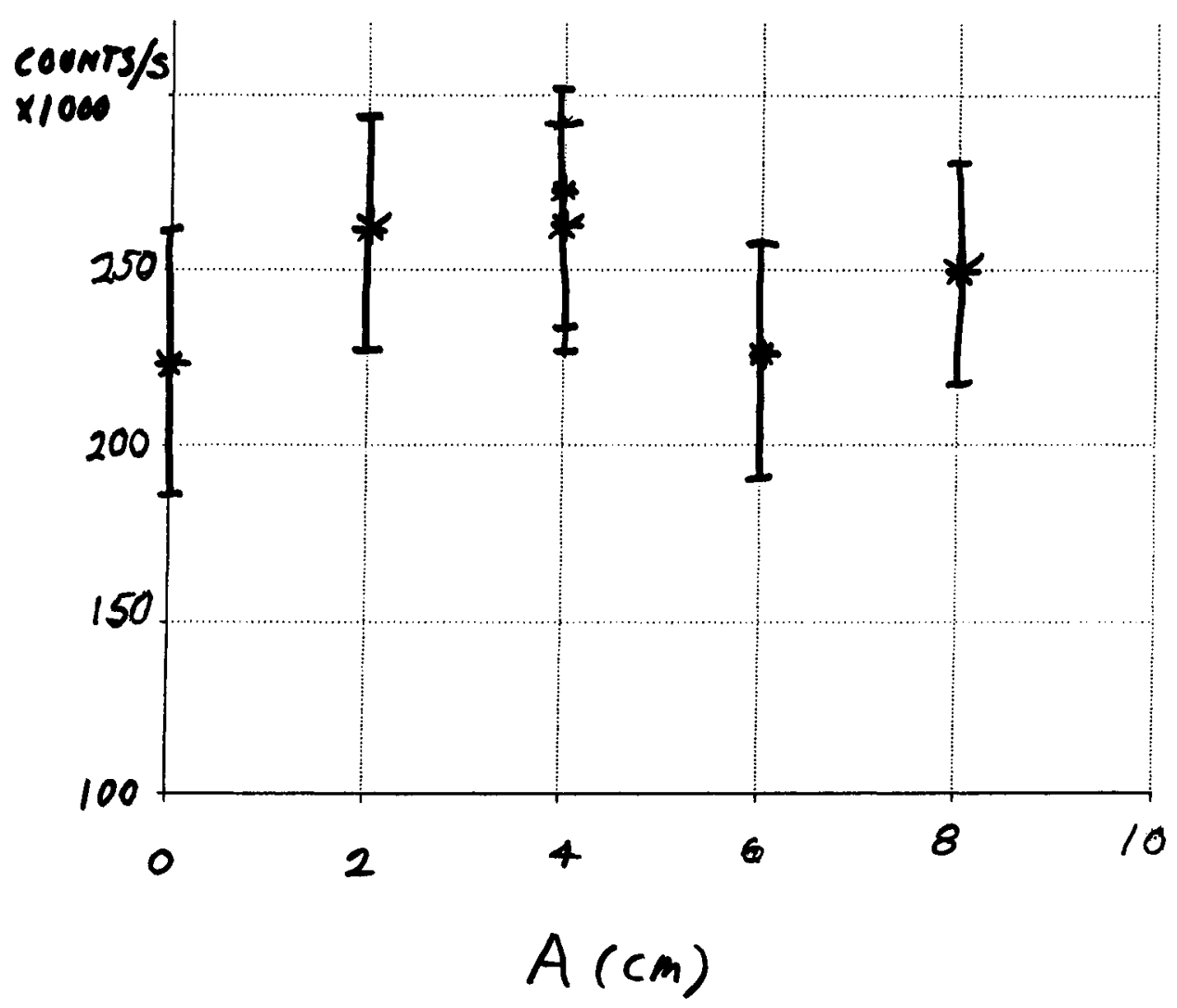

FIGURE 4. PMT response in Counts/S (photoelectron per second) vs. distance $A(\mathrm{~cm})$, the position of the LED measured from the PMT central axis. There is no significant change in the variation in counts as the position $A$ is varied. The data has been corrected for a small systematic effect $(15 \%)$ due to the distance variation of the LED from the PMT surface. The SIMION calculations and the experimental verification with a Hammamatsu phototube both indicate that a hemispherical emission geometry can be used to focus positrons in the proposed beam. 
As an additional check of the focusing predictions of the SIMION simulations, a transverse magnetic field was applied with a Helmholtz coil in the laboratory. The PMT response was compared to the SIMION result, with a similar magnetic field strength and geometry. Although the trajectories were quite complicated, there was an unmistakable similarity in the SIMION and the PMT measurement results. For example, the strength of the magnetic field to cut the photocurrent in half was measured to be $80 \mathrm{G}$ in the experiment and $95 \mathrm{G}$ for the SIMION simulations. This leads us to believe that the SIMION calculations are indeed reliable for this class of hemispherical geometries, and a diameter reduction of 50:1 has been demonstrated convincingly.

In the study of magnetic field effects, we found a small sensitivity to magnetic fields at the $\sim 3 \mathrm{G}$ level. Large diameter, low current coils could easily be incorporated to cancel stray fields, if they were of that magnitude.

The test of higher beam current was performed with a uniform light source illuminating the photocathode, and a United Detector Technology 40x opto-meter monitoring the irradiance $\left(\mathrm{W} / \mathrm{cm}^{2}\right)$. The estimated number of electrons leaving the surface was made using a photoefficiency of $\sim 15 \%$ given by the manufacturer for the wavelength of light used. With an illuminance of $100 \mathrm{nW} / \mathrm{cm}^{2}$ a current at the first dynode was measured directly to be $1.6 \mu \mathrm{A}$. The response was linear up to $10 \mu \mathrm{W} / \mathrm{cm}^{2}$.

In conclusion, the focusing without a grid seems to work well, and it appears to have many advantages over the design involving a grid. Barring any unforeseen problem, this concept will be incorporated into the ISPS beam in the demonstration Phase I beam. ${ }^{1}$ We are confident that the focusing, as predicted by SIMION for this class of gridless spherical geometries, will have a diameter reduction of 50:1.

1. See B. L. Brown elsewhere in this volume.

2. Grid focusing of positrons from a curved surface appears in Eric $\mathrm{H}$. Ottowitte, "High-Intensity Positron Beam Via $200 \mathrm{MW}$, Large Volume, Test (Fission) Reactor", Proceedings of the BB Factory Workshop, January 27, 1988 (World Press).

3. See H. Makowitz elsewhere in this volume.

4. A. Zecca and R. S. Brusa, Nuclear Inst. and Meth. in Phys. Research A313, 337(1992).

5. J. D. Jackson, Classical Electrodynamics, Vol 2, problem 3.11. 